\title{
Autonomous Mobile Robots Localization with Multiples iGPS Web Services
}

\author{
Camilo Christo, Edwin Carvalho, Miguel Pedro Silva and Carlos Cardeira \\ IDMEC/IST, Technical University of Lisbon \\ Av. Rovisco Pais, 1049-001 Lisboa, Portugal \\ \{cchristo, miguel.silva, cardeira\}@ dem.ist.utl.pt, edwin.carvalho@gmail.com
}

\begin{abstract}
Diverse systems have been proposed to aid the navigation of mobile robots in indoor environments. Simultaneously, communication technologies that allow the integration of diverse systems even when these are developed on different platforms, have been proposed.

The indoor Global Position Systems (iGPS) Web Service architecture proposed by Christo [7] joins the emerging standard Web Services technologies with the iGPS idea to allow the integration of mobile robots in indoor environments in a fast and easy way.

In this paper, we suggest an architecture for the use of multiples iGPS Web Services for mobile robots localization. To emphasize the usefulness of the multiple iGPS web services localization system, an application of localization of mobile robots is presented.
\end{abstract}

\section{Introduction}

In order to correctly move in any non-structured environment, an Autonomous Mobile Robot (AMR) requires a robust navigation system. Traditionally a variety of sensors such as rotary encoders, laser range finders, infra-red sensors and ultrasonic sensors were used by AMR's to locate themselves and to avoid obstacles in their proximity. However the great computational burden placed on the processing units by all the collected data, analysis, and the fact that the navigational capabilities of the robots remain limited since the AMR still does not have an overview of the entire environment makes the use of such technologies inadequate for many applications. A system that can locate AMR's and possible obstacles and relay the information to the AMR's is therefore important.

To overcome the localization limitation, several systems have been researched. For example, the Cricket Indoor Location [5] which relies on active beacons placed in the environment. These beacons transmit simultaneously two signals (a RF and an ultrasound wave). Passive listeners mounted, for example, on AMRs can, by knowing the difference in propagation speed of the RF and ultrasound signals, estimate their own position in the environment.

GSM and WLAN technologies can also be used for localization. By using triangulation methods and measuring several signal parameters such as the signal's angle and time of arrival, it becomes possible to estimate the position of a mobile transmitter/receiver in the environment [6]. However, these systems are still imprecise, therefore, are not currently adequate for mobile robot localization.

Vision based localization systems present several interesting advantages over competing solutions. Since these systems use cameras placed throughout the environment, no specialized electronic devices have to be installed on the AMRs. For instance, indoor Global Positioning Systems (iGPS) and Intelligent Spaces for mobile robot localization and control inside buildings have received widespread attention in recent years $[1,2,3]$.

With iGPS and Intelligent Spaces, AMRs and obstacles can be located in a given environment however, the integration of new AMRs in the aforementioned systems can be a difficult task because a common and standard method of communication does not exist which, in turn, implies that an AMR integrated in a particular network cannot immediately be used in another network.

The Service Oriented Architecture (SOA) approach defines relationships between service requesters and service providers having no direct association to the software language or operating systems, hence allowing seamless communication between different devices and systems. One popular technology which has been used to develop systems with a SOA is the Web Service technology. Web Services allow well-defined access to service interfaces which may facilitate integration of new agents (such as mobile robots) and intelligent mechatronic devices in any given environment and for distributed intelligence $[10,11,16]$. As an example, in [4] virtualization is proposed as a way to integrate resources inside enterprises. In other words, each resource (devices, processes, etc.) is virtualized as a Web Service to be able to offer their functionalities inside a standard Web environment.

The present paper attempts to emphasize the advantages of iGPS systems surrounded by a standard Web interface (the iGPS WS: indoor Global Position System Web Service) such as that presented by Christo in [7]. In order to easily integrate new iGPS localization systems and new mobile robots in an environment, the concept of a Global iGPS Database and Global Pattern Database is presented. The Global iGPS Database is a Web Service where the ex- 
isting iGPS WS addresses can be registered to allow latter consultation by AMRs or other applications. On the other hand, the Global Pattern Database is another Web Service where the mobile robots register their identification patterns in order to allow their identification by the iGPS units placed in the environment. Finally, an architecture for the use of multiple iGPS WS is presented, in order to allow an open and objective way for the localization of mobile robots in indoor environments.

This paper is organized as follows: In Section 2 an overview and description of the iGPS Web Services is done. In Section 3, underlying principles for an architecture for the use of multiple iGPS Web Services are presented, as well the Global iGPS and Pattern database concepts. Section 4 presents the technologies used on the practical implementation of the concepts and ideas previously exposed. Section 5 presents some practical results obtained through an implementation of the described architecture for localization of mobile robots with multiples iGPS WS and a tracking trajectory application is also presented. Finally, in Section 6, are the conclusions and some possible future research areas are presented.

\section{2. iGPS Web Service Overview}

Web Services are dependant on open standards such as the eXtensible Markup Language (XML) and the HyperText Transfer Protocol (HTTP) and not depend on any particular operating system, which make them an excellent option as a basis for SOA (see [9] and the references therein).

\section{1 iGPS Web Service Concepts}

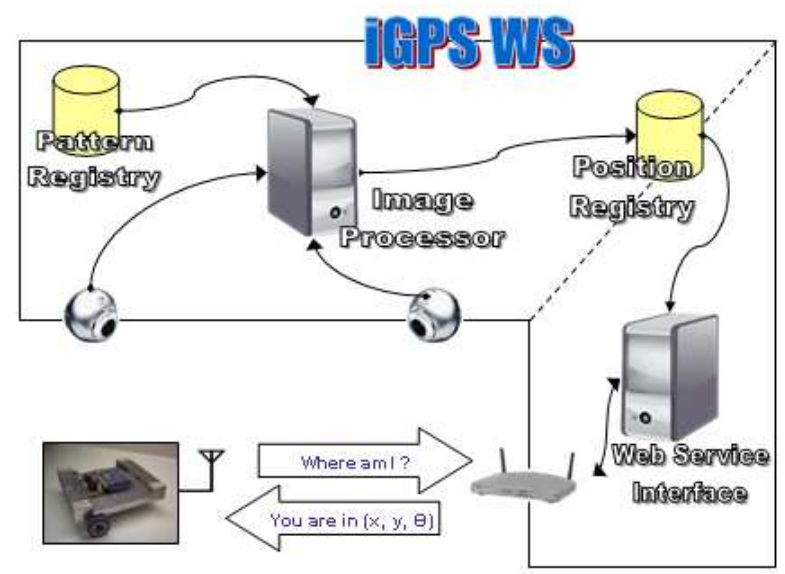

Figure 1. iGPS Web Service [7, 12].

By introducing a standard Web Service interface between a traditional iGPS and clients of iGPS services (Fig. 1), information regarding positioning and orientation of robots in the environment can be made available in a seamless, transparent form. The iGPS system can be based on any methodology described in the introduction of this paper, however, vision based iGPS systems are appropriate for robot tracking and obstacle identification since the identified objects do not need to be equipped with any special device and the position estimation does not involve any form of contact between the iGPS and the mobile robots.

However, vision-based iGPS needs some form of identification pattern (e.g. SIFT Keypoints) to distinguish an AMR from another [12], hence a iGPS needs to have knowledge of the AMR that are present in the environment and their respective identification patterns. The iGPS should also know when an AMR is leaving or entering its environment so it can decide if it should search for that particular identification pattern. The most transparent way of doing this is by AMR's notification or by a client application which automatically informs the iGPS system that a certain AMR is entering (or leaving) the environment and its identification pattern.

To facilitate access by outside agents for the position of the AMRs, the iGPS records in a Position Registry database the current position of each AMR. The Web Service interface wrapped around the iGPS application accesses the database and returns the position of the robot when inquired by an outside client application (e.g., traffic control application).

\section{2 iGPS Web Service Architecture}

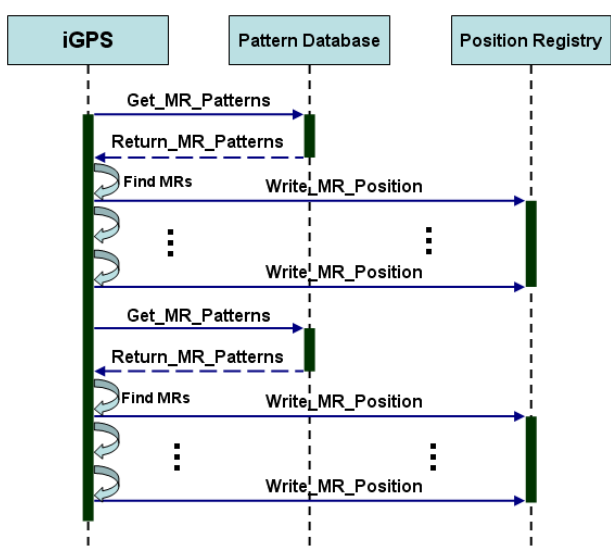

Figure 2. iGPS application [7, 12].

Figure 2 represents the iGPS application process and the communication with the pattern database and Position Registry database throughout time. When the iGPS application initializes, it first attempts to verify which AMRs are currently in the environment. Through the internal function Get_MR_Patterns, the iGPS asks the pattern database for the registered AMRs and their respective identification patterns. After receiving the data concerning the AMRs to locate, the iGPS begins searching for the registered AMRs and estimate their position in the environment. When the iGPS correctly locate an AMR, it uses a Write_MR_Position function to update the SQL position 
database. If the data is time relevant, the information may be time stamped [7].

The vision-based iGPS tries to update the Position Registry at the camera's frame rate to give a refresh position data. At a lowest frequency, the iGPS application also updates its tracking system by accessing the pattern database to only search for patterns that correspond to AMRs which are, at that instance, in the environment.

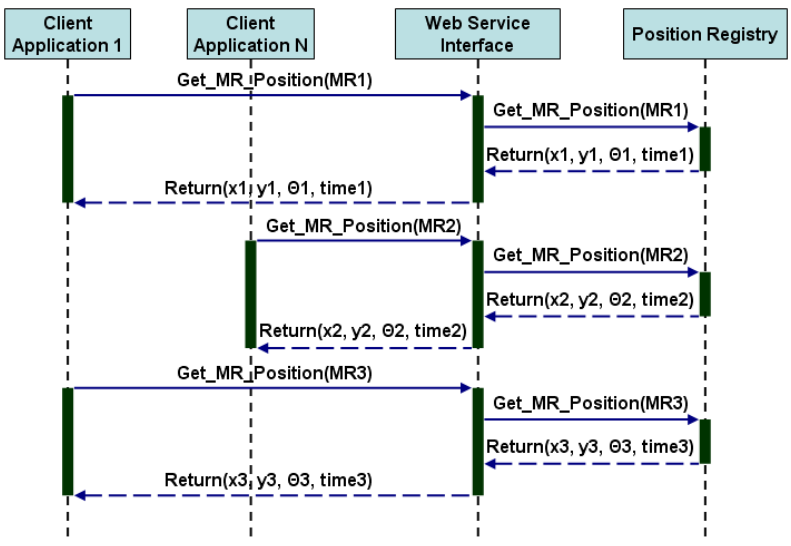

Figure 3. iGPS WS interface $[7,12]$.

In Fig. 3, the inquiry process regarding the location of an AMR using Web Services is presented. Several applications can ask, at any given time by using the corresponding Web Service and the Get_MR_Position Web method, for the estimated position of a AMR by adding the mobile robot's identifier. The iGPS WS will return the estimated position coordinates state of the inquired mobile robot in the environment. The data regarding the robots localization obtained through the iGPS WS can be fused with odometry data to improve the accuracy of the information. Other clients (e.g., Client Application 1) can inquire the iGPS WS for tracking purposes, for example to create data logs with the paths executed by different mobile robots [7]. A "NULL" response shall be returned if the iGPS does not locate the respective mobile robot.

\section{Multiple iGPS Web Services Architecture}

\section{1 iGPS WS and AMRs Registration}

Since each AMR and iGPS WS can be added or removed from the environment, it is not practical to inform each AMR (or iGPS WS) when an iGPS WS (or AMR) is added to (or removed from) the environment.

The easiest workaround for the above described problem is to create two independent databases each of which can be accessed using Web Services. The first could act as an AMR registry database (containing each AMRs identification name, identification pattern, etc.) and the second database should store the iGPS WS registry information (identification name, Web address, etc.).

\subsection{1 iGPS WS Registry (Global iGPS Database)}

Figure 4 schematically presents the iGPS WS registry process in a given environment. Every time a new iGPS WS is introduced in the environment, it shall register itself on the Global iGPS Database by using a Web Service whose address is public. When the iGPS WS is deactivated, it uses the same Web Service to remove its registry. The AMRs in the environment can know what iGPS WS are activated at any instant by accessing the aforementioned Global iGPS database.

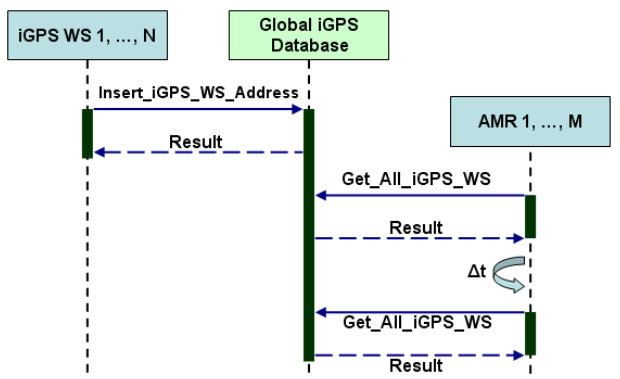

Figure 4. Global iGPS Database.

\subsubsection{AMR Registry (Global Pattern Database)}

As presented for the iGPS WS, the AMRs, when introduced in the environment, register themselves on an AMR registry database (the Global Pattern Database, see Fig. 5) which can be accessed through a Web Service whose address is known beforehand. The active iGPS WS can access the pattern database to verify what robots are currently in the environment and how to identify them.

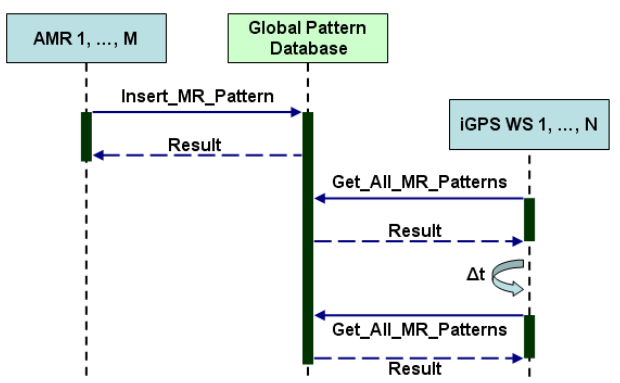

Figure 5. Global Pattern Database.

\section{2 iGPS WS Units Distribution}

Since mobile robots can move throughout cluttered rooms and cover great areas, one camera is not, normally, sufficient to cover the entire area in which the AMR will move. On the other hand, connecting several camera's to a unique processing unit is not plausible due to the computation burden that image processing implies which may consequently break the system. However, an option is to use individual units distributed throughout the several rooms that compose the AMR's workspace (Fig. 6). In this system, each unit will have its own designated area in which it is responsible for the localization of AMRs. Each 
of the mentioned before units will have their own Position Registry in which they record relevant localization information.

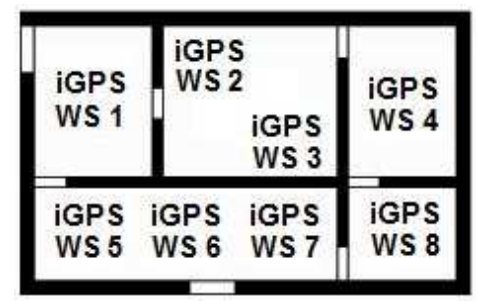

Figure 6. Example of iGPS WS units distribution [7].

\subsection{Concurrent iGPS}

Individual iGPS WS units can be placed in the environment. Through the associated iGPS application each unit make the search of mobile robots in some area and has access to the Global Pattern Database to update the mobile robots to find (Fig. 7).

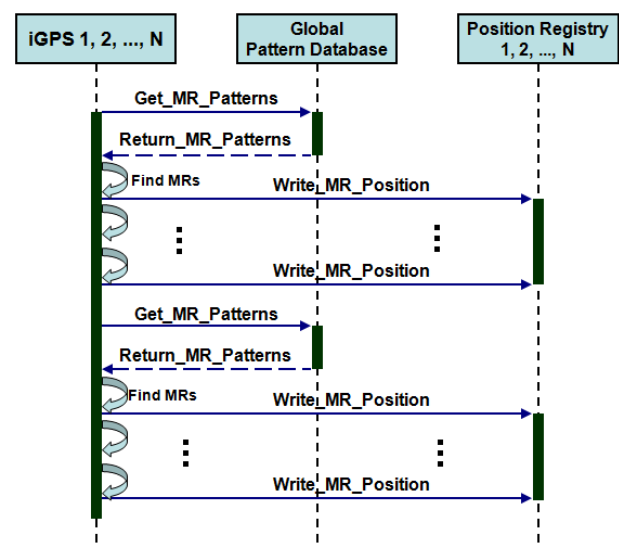

Figure 7. Concurrent iGPS [7].

Each iGPS application possesses its own Position Registry database where the position of each robot will be kept and continuously updated. If two or more iGPS cover the same area, then the information regarding all the AMRs in that area will be stored and updated by all the iGPS that are viewing the AMRs in their personal Position Registry database.

\subsection{Switching among iGPS Web Services}

Each iGPS WS makes available several Web methods accessible anywhere and by any client application. The commutation of iGPS WS can easily be done by changing the Web Service interface address (Fig. 8). As previously referred, by spreading iGPS systems and the corresponding iGPS Web Services throughout the environment, larger areas can be surveyed, hence, allowing AMRs to navigate freely throughout greater environments. However, in order to accomplish this, the AMRs need to know how many iGPS are active in the environment and the Web addresses of their interfaces (see section 3.1.2). If necessary, the AMRs should have access to information regarding the area covered by each iGPS WS module.

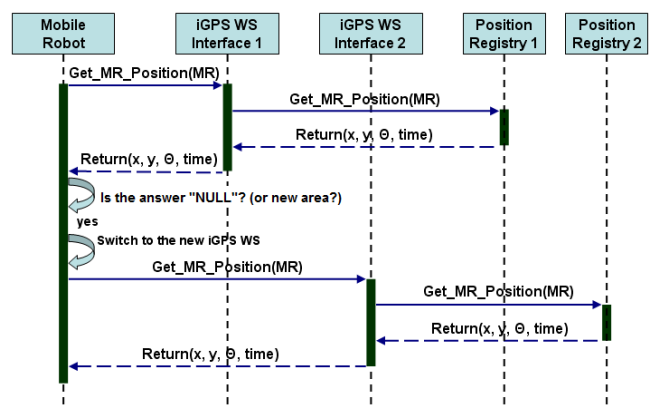

Figure 8. Switching among iGPS WS [7].

Once the Web addresses of each active iGPS WS are known, the AMRs start the localization process by successively asking each iGPS WS for its own coordinates. The AMR continues this process until it obtains a positive answer and from then onwards, it continues connected to that iGPS WS until the iGPS stops locating the AMR. When such happens, it restarts the aforementioned localization process until it finds a new iGPS WS that is currently locating the AMR. If associated to the iGPS's Web addresses, information regarding the covered area of each iGPS is provided, the AMRs may directly question the iGPS WS of the area in which it currently is instead of asking all iGPS WS.

In the following sections, results that validate the use of multiple iGPS WS as a way of integrating and locating mobile robots in closed environments by resorting to standard Web interfaces is presented.

\section{Multiple iGPS WS: Implementation}

\subsection{Experimental Setup}

In order to properly test the use of Web Services and iGPS for mobile robot localization, the following setup was developed (see Fig. 9).

Low cost mobile robots [13], were changed to allow easy recognition by the iGPS WS. The changes to these mobile robots consist on placing a unique pattern that can be identified by image processing.

Also three iGPS WS units were placed in the environment. Each unit consists of a camera and a computer (Intel Pentium 4, CPU $3.00 \mathrm{GHz}$, GB RAM) responsible for the image processing and for storing the iGPS Position Registry database. The afore mentioned Position Registry is a SQL database managed by MS SQL Server 2005 and the Web Service interface between the SQL database and the client applications was built using ASP.NET 2.0. The communication among units (iGPS WS, AMRs) is made by a WLAN (Wireless Local Area Network, 54 Mbps). 


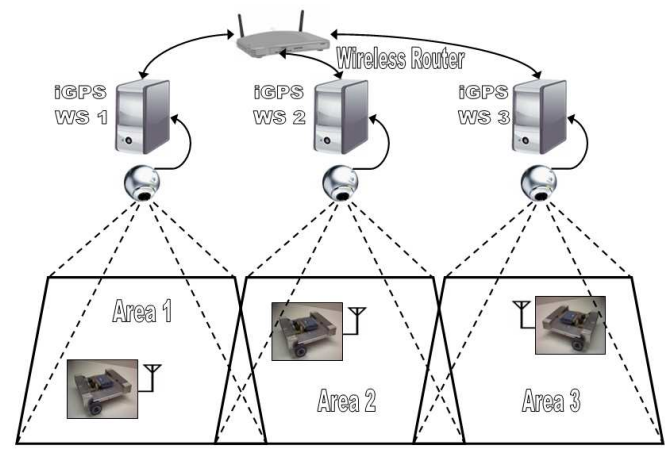

Figure 9. Experimental Setup.

\section{2 iGPS Application based on the HSV colorspace}

Several vision methods may be used to detect a mobile robot in the environment. For instance, Scaleinvariant feature transform (SIFT) have been suggested in $[12,14,15]$ to recognize a mobile robot by comparing the sift keypoints of the image being captured by the iGPS system with keypoints stored in the pattern database. Another method to locate robots in the environment consists on using color bars placed on each mobile robot and comparing the parameters of the color band to those stored in the pattern database (see [7, 8] and the references therein).

In this paper, the latter option was preferred. Color targets of different colors were placed on the AMRs and the HSV (hue, saturation and value) bounds of each color targets were used as a mobile robot identifier. The choice of the HSV colorspace to locate the AMRs is related to this colorspace's invariance to lighting changes. However an application based on RGB or another color space is also possible and can be implemented.

The iGPS application identifies each AMR by, first of all, accessing the Global Pattern database to obtain the HSV bounds of each color target placed in the mobile robots. Next, by thresholding the images from the cameras in order to obtain the aforementioned HSV parameters, and calculating the geometric center of the thresholded image, the position of each mobile robot can be estimated in a global cartesian frame using projection equations.

\section{Experimental Results}

The vision system is an important piece of the iGPS in respect to the Position Registry frequency update. Since the vision system is out of scope of the present work, it is important to define the iGPS WS capabilities, with respect to the update frequency of the Position Registry database. The results presented in section 5.1 concern the writing capabilities of the iGPS application and the reading capabilities of the AMRs, assuming that the vision system has no time delay (there is no image processing). Section 5.2 presents the results considering in addition the implemented vision system based on HSV. An example of the tracking of two mobile robots by resorting to in- formation obtained by three iGPS WS units placed in the environment is presented in section 5.3.

\subsection{Update and Accessing Frequency of the Position Registry Without the Vision System Limitation}

The results presented in this section are obtained by writing random position values in the Position Registry database at the maximal possible rate. Virtual client applications (virtual AMRs) uses the WLAN to invoke the Get_MR_Position Web method, in order to obtain the different Position Registry values.

The first iGPS WS test consists of writing random positions values of one virtual AMR in the Position Registry Database. At the same time one virtual AMR uses the WLAN to inquire at the maximal possible rate the iGPS WS for its position. The next step is obtain the position of the same virtual robot using two and three different virtual client applications.

The second test consists in writing two random positions values of two virtual AMR. In a first step we have one application asking the iGPS WS for each AMR position (two applications in total). The second step consists in having two applications asking for each AMR position (four applications in the total) and in the third step three different applications are asking for each AMR position (six applications in total).

Finally, the third test consists in writing three random values in the Position Registry Database to simulate three different AMRs. In a first step we have one application asking each AMR position (three applications in total). The second step consists in having two applications asking for each AMR position (six applications in the total) and finally in the third step three applications are asking for each AMR position (nine applications in total).

Each table value is the average of three experiments of 180 seconds each.

\subsubsection{Position Registry Updating Frequency}

\begin{tabular}{|c|c|c|c|c|}
\cline { 3 - 5 } \multicolumn{2}{c|}{} & \multicolumn{3}{c|}{ \# of Readings by Position } \\
\cline { 3 - 5 } \multicolumn{2}{c|}{} & 1 & 2 & 3 \\
\hline Updated & 1 & 63.98 & 63.98 & 63.58 \\
\cline { 2 - 5 } Virtual & 2 & 63.98 & 63.60 & 63.02 \\
\cline { 2 - 5 } Positions & 3 & 61.42 & 49.73 & 35.79 \\
\hline
\end{tabular}

Table 1. PR Update frequency $[\mathrm{Hz}]$.

Table 1 shows the capability of the iGPS application when it comes to the updating of the Position Registry database without the image processing limitation.

When a iGPS unit makes the update of one or two AMRs (first and second line of the table), the update capabilities of the Position Registry is over $63 \mathrm{~Hz}$, independently of the number of applications accessing the position database. When the iGPS is updating three positions in the database, its updating frequency depends on the 
number of external applications reading each robot position at the same time. The worst case scenario happens when the iGPS is updating the position of the three mobile robots in the registry and a total of nine external applications (three per position) are inquiring the database. In this case, the update frequency decreases to $35.79 \mathrm{~Hz}$.

\subsubsection{Position Registry Access Frequency}

\begin{tabular}{|c|c|c|c|c|}
\cline { 3 - 5 } \multicolumn{2}{c|}{} & \multicolumn{3}{c|}{ \# of Readings by Position } \\
\cline { 3 - 5 } \multicolumn{1}{c|}{} & 1 & 2 & 3 \\
\hline Updated & 1 & 44.93 & 62.67 & 62.50 \\
\cline { 2 - 5 } Virtual & 2 & 63.05 & 57.73 & 49.40 \\
\cline { 2 - 5 } Positions & 3 & 62.84 & 49.15 & 38.40 \\
\hline
\end{tabular}

Table 2. PR Access frequency $[\mathrm{Hz}]$.

Table 2 shows that when consulting the Position Registry database through the Web Service interface no significant reductions in the frequency of the mobile robots position answers are implied.

The worst achieved result $(38.4 \mathrm{~Hz})$ happens when three positions are updated in the Position Registry and, simultaneously, nine applications accesses the database for reading operations (three per mobile robot).

Since the goal of the iGPS is aiding in the navigation of the mobile robots (by correcting odometry errors and by locating other mobile robots in the environment), update frequencies of $38 \mathrm{~Hz}$ can be considered sufficient.

\subsection{Update and Accessing Frequency of the Position Registry With the Vision System Limitation}

In this section, the experimental results with the HSV image processing unit are presented. The procedure to obtain the results is identical to the one presented in section 5.1.

\subsubsection{Position Registry Updating Frequency}

\begin{tabular}{|c|c|c|c|c|}
\hline & \multicolumn{3}{|c|}{ \# of Readings by Position } \\
\hline & & 1 & 2 & 3 \\
\hline \multirow{3}{*}{$\begin{array}{c}\text { Updated } \\
\text { AMR } \\
\text { Positions }\end{array}$} & 1 & 44.20 & 41.55 & 39.90 \\
\hline & 2 & 23.31 & 15.37 & 11.79 \\
\hline & 3 & 13.98 & 9.13 & 7.25 \\
\hline
\end{tabular}

Table 3. PR Update frequency [Hz].

The ability to update the Position Registry strongly decreases with the number of robots to locate and the number of outside applications accessing the positions (see Table 3 ). This decrease in update frequency was expected since the image processing takes a considerable computation time and also because the computer resources are being shared by the SQL and the iGPS application.

\begin{tabular}{|c|c|c|c|c|}
\cline { 3 - 5 } \multicolumn{2}{c|}{} & \multicolumn{4}{|c|}{ \# of Readings by Position } \\
\cline { 2 - 5 } \multicolumn{2}{c|}{} & 1 & 2 & 3 \\
\hline Updated & 1 & 49.13 & 63.48 & 62.64 \\
\cline { 2 - 5 } AMR & 2 & 62.35 & 59.60 & 48.76 \\
\cline { 2 - 5 } & 3 & 62.75 & 47.60 & 34.27 \\
\hline Positions
\end{tabular}

Table 4. PR Access frequency $[\mathrm{Hz}]$.

\subsubsection{Position Registry Access Frequency}

The access frequency values to the Position Registry by the exterior applications are similar that presented in section 5.1.2, since they do not depend on the update frequency of the position database.

\subsection{Tracking Trajectories}

The advantages of the iGPS WS systems can be demonstrated by Fig. 10. In the aforementioned figure, the trajectories of two robots in an indoor environment under supervision of three iGPS WS units are presented. It is easy to verify that the use of three iGPS WS units is advantageous since it allows the localization of the different robots even when there is a transition between iGPS WS due to their covered areas (see Fig. 9).

The developed tracking application automatically obtains the addresses of each iGPS WS unit through the Global iGPS Database. Everytime the tracking application can not obtain the position of a mobile robot ("NULL" answer), the tracking application asks the following iGPS WS unit registered in the database (switches between units) by using the method described in section 3.4.

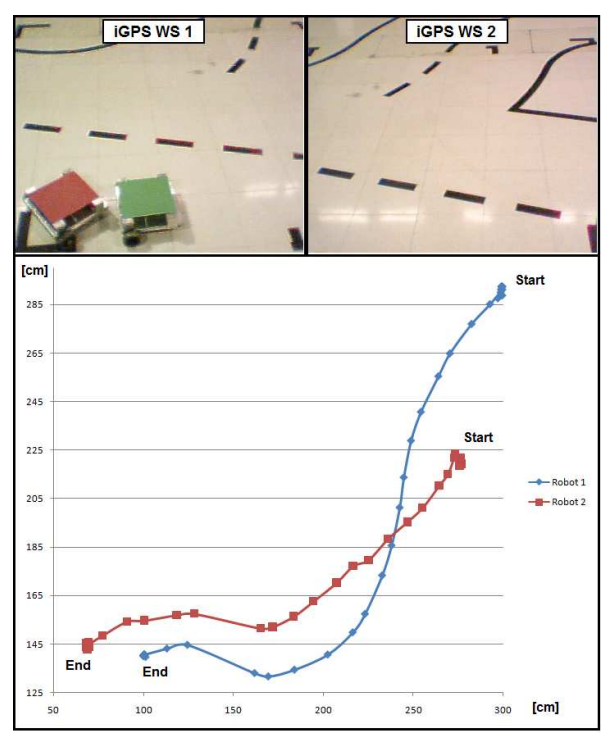

Figure 10. Position Tracking. 


\section{Conclusions and Future Work}

\subsection{Conclusions}

The presented system is modular since it allows the insertion/removal of iGPS units and simultaneously the insertion/removal of mobile robots in the environment without having to reconfigure the entire system. Since the communication protocol is based on open standards, it shall be possible, in the future, to add other iGPS units or mobile robots even when their software was developed in another programming language or runs on another operating system.

The addition of auxiliary web services (Global iGPS Database, Global Pattern Database) is advantageous in practical implementation for a multiple iGPS localization system, since it becomes necessary to inform existing iGPS units and mobile robots of only two web addresses, independently of the number of iGPS units and mobile robots operating in the environment. If for robustness, it becomes necessary to replicate the Global iGPS database and the Global pattern database in the environment, it will, forcefully, become necessary to inform the iGPS units and the robots of all the web addresses.

Other tests not presented in this paper shown that it is possible to inquire the iGPS WS for eight different positions through eight different applications with a frequency of approximately $25 \mathrm{~Hz}$. However, when the iGPS is writing eight random positions in the Position Registry Database, the frequency is below $10 \mathrm{~Hz}$.

The experimental results obtained are satisfactory but demonstrate that there are two areas of improvement. Firstly, the vision system and secondly, the position registry. Although the vision system is not the focus of this work, it is essential for the end results of this research since, without a fast and robust vision system, the localization system does not function correctly. Significant improvements in the vision system can be achieved if the robot location and decision is made by computing the Mahalanobis distance between the acquired image pixels and the previously saved mean and covariation matrices of the original robot pixels. Regarding the position registry, its update frequency should be improved to allow faster access from exterior applications. In order to accomplish this, the iGPS system should be tested with other databases to test the ability of these databases to constantly manage the flow of data (real time databases).

\subsection{Future Work}

As future work we intend to:

- Increase the robustness of the iGPS application: The vision system plays an essential role in the localization of the mobile robots, hence needs to be optimized for speed and must be invariant to external disturbances present in the environment;

- Increase the update frequency of the mobile robot Position: By eliminating auxiliary management ser- vices (such as the SQL database), storing the localization information inside the iGPS application and sharing that information using Web Services, faster update frequencies should become possible;

- Implement the iGPS WS ideas in Flexible Manufacturing Cells (FMC): The main idea is to create an intelligent FMC where machines, mobile robots and the environment, are able to communicate using standard interfaces.

\section{Acknowledgments}

This work was partly funded by the FCT through program POCI 2010 - 10-46 - IDMEC, subsidized by FEDER.

\section{References}

[1] Y. Hada and K. Takase, "Multiple Mobile Robot Navigation Using the Indoor Global Positioning System (iGPS)", Proc. of the 2001 IEEE/RSJ Int. Conf. on Intelligent Robots and Systems, vol. 2, pp. 1005-1010, Nov. 2001.

[2] Y. Hada et al, "Trajectory Tracking Control of a NonHolonomic Mobile Robot using iGPS and Odometry", Proc. of IEEE Int. Conf. on Multisensor Fusion and Integration for Intelligent Systems3, pp. 51-57, Aug. 2003.

[3] J.-H. Lee and H. Hashimoto, "Controlling mobile robots in distributed intelligent sensor network", IEEE Trans. on Ind. Electronics, vol. 50, no. 5, pp. 890-902, Oct. 2003.

[4] R.S. Kumar et al, "Virtualization for Manufacturing Web Services: a WS-RF approach", Int. Journal of Information Technology, vol. 11, no. 3, pp. 40-51, 2005.

[5] N. B. Priyantha et al, "The Cricket location-support system", Proc. of the 6th annual international conference on Mobile computing and networking, pp. 32-43, 2000.

[6] A.H. Sayed, A. Tarighat and N. Khajehnouri, "NetworkBased Wireless Location: Challenges faced in developing techniques for accurate wireless location information”, IEEE Signal Processing Magazine, vol. 22, no. 4, July 2005.

[7] C. Christo, "Service Oriented Architecture for Mobile Robots Integration", Msc Thesis, Universidade Técnica de Lisboa, Instituto Superior Técnico, 2008.

[8] Edwin Carvalho, "Localization and Cooperation of Mobile robots Applied to Formation Control", Msc Thesis, Universidade Técnica de Lisboa, Instituto Superior Técnico, 2008.

[9] C. Christo and C. Cardeira, "Plug-and-Play Autonomous Mobile Robot (P\&PAMR)”, Proc. IEEE Int. Symposium on Ind. Electronics, ISIE 2007, pp. 3215-3220, June 2007.

[10] C. Christo and C. Cardeira, "Trends in Intelligent Manufacturing Systems", Proc. IEEE Int. Symposium on Ind. Electronics, ISIE 2007, pp. 3209-3214, June 2007.

[11] C. Christo and C. Cardeira, "Service Oriented Architecture for Mobile Robot Localization", Proc. 12th IEEE Conference on Emerging Technologies and Factory Automation, ETFA 2007, pp. 888-891, Sept. 2007.

[12] N. Barral, C. Christo and C. Cardeira, "iGPS Web Service based on SIFT for Mobile Robot Localization”, Proc. 8th Portuguese Conference on Automatic Control, CONTROLO'2008, pp. 548-553, July 2008. 
[13] C. Cardeira and J.S. da Costa, "A low cost mobile robot for engineering education", IECON 2005, 32nd Annual Conference of IEEE Industrial Electronics Society, Nov. 2005.

[14] D.G. Lowe, "Object recognition from local scale-invariant features", International Conference on Computer Vision, pp. 1150-1157, 1999.

[15] D.G. Lowe et al., "SIFT-ing Through Features with ViPR", IEEE Robotics \& Autom. Magazine, pp. 72-77, 2006.

[16] J. Lastra and I. Delamer, "Semantic Web Services in Factory Automation: Fundamental Insights and Research RoadMap”, IEEE Trans. Ind. Inf., vol. 2, no. 1, Feb. 2006. 